\title{
Numerical Model to Predict Wax Crystal Size Distribution in Solvent Dewaxing Unit
}

\author{
Nassir D. Mokhlif, Hussain H. Al-Kayiem, and Masri Bin Baharom
}

\begin{abstract}
A mathematical model was implemented to predict the wax crystal size distribution of distillate lube oil SN500. The model solved the coupled equations for the heat transfer and moments of the population balance numerically. The predicted temperatures and wax crystal size distributions were validated with actual unit database and experimental measurements. The kinetics of the wax crystallization considered only the nucleation and crystal growth. $\mathrm{N}$-alkanes from $n-C_{22}$ to $n-C_{36}$ were tested to select compound for representation of the wax fraction. The nucleation process order constant was fitted from wax recovery experimental measurements. The developed numerical model was proved to be capable to predict wax crystal size distribution in real solvent dewaxing plant. The model results were found to be in good agreement with the process data.
\end{abstract}

Index Terms-Solvent dewaxing, numerical model, wax crystal size distribution, lube oil production.

\section{INTRODUCTION}

The wax is probably the most troublesome product in the lube oil manufacturing. Its presence in lubricating oils prevents free movement at lower temperatures [1]. One of the separation techniques to remove the wax fraction is done by refrigeration to crystallize the wax and solvent to dilute the oil portion. Solvent dewaxing process is selectively removes higher $\mathrm{n}$-paraffins that are crystallize easily to form the wax rather the non-linear paraffins which crystallize very solwly [2], [3]. The lube oil feedstock and solvent mixture was chilled at a specified cooling rate in a series of hairpin double pipe scraped surface heat exchangers (DPSSHE's) and chillers (DPSSC's). The current solvent dewaxing unit was the most widely method used to separate the wax from the waxy feedstock oils. The unit can handle different types of distillate lubricating oil and residual fractions with a control system allows variation in the dilution ratio of the feedstock to the solvent in optimum range without affecting the cooling rate and degree of supersaturated concentrations of the flow.

The widely solvent composition used in solvent dewaxing process consists of aromatic compound (Toluene) and Ketone compound (MEK). The Toluene as a single component is capable of increasing the oil solubility at crystallization temperature and to reduce the viscosity of oilsolvent solution.

The MEK is a poor solvent power for wax and relatively have good solvency for the oil portion. In the filtration section the oil is still soluble in solvent mixture while the

Manuscript received August 4, 2013; revised October 2, 2013.

The authors are with the Mechanical Engineering Department, UTP, Tronoh 31750, Perak, Malaysia (e-mail: nassirdhamin@yahoo.com). wax is not. The advantage of the crystallization process as a separation technique is relatively demand low energy with high purity of the product.

In addition of the performance analysis of the DPSSE as heat transfer equipment by using the conservation equations such as the heat transfer, mass or momentum (mainly fluid flow), design this instrument as a crystallizer in particulate system involved further transport formula to describe the crystals characteristics. This transport equation known as population balance equation (PBE) required accounting the crystals number, and crystal size distribution. Also, this equation quantifies processes such as nucleation, crystal growth, aggregation, and breakage and so on. The difficulties facing the designers of the industrial SSHE as a crystallizer arising from the scarcity of the experimental data for the individual processes rather than the limited and unreliability of these published data. The mathematical models of crystallization process in SSHE essentially, the ice crystallization from scours solution, sorbet, and ice cream processes, the description of the crystallization kinetics limited to the nucleation (homogenous or heterogeneous), crystal growth, and the breakage in some studies.

In general the studies referring to develop a mathematical model of coupling the heat transfer, fluid flow, and the kinetics crystallization for the separation technique in scraped heat exchanger (SSHE) are limited. These studies introduced a numerical simulation of the fluid flow and heat transfer combined with the population balance to represent the kinetics of ice particles crystallization using commercial CFD software [4], [5]. Represent the wax crystallization characteristics in real solvent dewaxing unit is quite difficult for unclassified mixture composition compare with the others crystallization processes that experimentally implemented for define concentration. In addition the experimental information data facing a shortage of the published researches and the kinetics crystallization information compare with e.g. ice crystallization process.

The publication of the modeling analysis in the dewaxing lube oil feedstock in SSHE for the previous studies was found to be very limited. Bessarabov et al. [6], proposed an analytical model (1-D) transient of the dewaxing process from a raffinate-solvent solution, the authors suggested that the temperature distribution of accumulated wax layer on pipe wall between two adjacent rotational scrape blades actions can be solved combined with wax crystallization kinetics. The kinetics of crystallization processes is described by using $\mathrm{j}^{\text {th }}$ moment transformation of population density function. A suggestion that the wax crystallized on the inside tube wall in scraped channel has the form of a hollow cylinder layer, so in order to solve the kinetic problem of wax crystallization in such a way that the 
temperature distribution in a wax layer crystallized on the tube wall at any moment of time must be determined. A numerical model developed by Jun Li et al. [7] to predict the wax crystal distribution (CSD) in solvent dewaxing. The theoretical model improved for one dimensional parallel flow direction in steady state condition. The authors used a pilot plant consists of four single elements of DPSSE s with multi solvent dilution points after each exchanger. The operation condition including the linear temperatures distributions, solvent composition, wax compound, and the crystallizer dimensions were examined. The results discussed the effect of operational condition on the wax CSD in the wax-oil-solvent solution. The author s tested three linear n-paraffins $\mathrm{C}_{24}, \mathrm{C}_{28}$, and $\mathrm{C}_{32}$ in order to determine the most appropriate component to represent the wax solid phase. This issue supported by the wax recovery in different operation conditions.

The main aim of the present paper is to develop a mathematical model to predict the wax CSD and the wax crystallization characteristics of lube oil feedstock SN500 in a series of DPSSE $s$ and DPSSC $s$ in solvent dewaxing unit. The validation implemented for current model compare with experimental CSD measurement of the filtrated slack wax.

\section{THEORETICAL MODELS FORMULATION}

\section{A. Energy Balance Equations in Exchangers}

The SSHE s performed as a counter current system, hence, in order to simulate the double pipe SSHEs numerically; the model equations for heat transfer balance for hot fluid (feedstock/solvent) in scraped channel and for differential segment $d z$ in steady state condition are as follows:

$$
\frac{d T_{h}}{d z}=-\frac{U P}{\left(n \& c_{p}\right)_{h}}\left(T_{h}-T_{c}\right)
$$

where $\kappa_{h}, C_{p_{h}}, U, P, T_{h}$ and $T_{c}$ are the hot fluid mass flow rate, heat capacity, overall heat transfer coefficient, scraper channel perimeter, hot fluid temperature and cold fluid temperature respectively. The differential equation for the cold fluid (filtrate) side is as follows:

$$
\frac{d T_{c}}{d z}=\frac{U P}{\left(n \& c_{p}\right)_{c}}\left(T_{h}-T_{c}\right)
$$

The energy balance in the chillers can be represented by replacing the coolant temperature $T_{c}$ in the Eq. (2) with the wall temperature of the outer diameter of the scraper channel with assumption of constant saturation temperature of the evaporated refrigerant in chillers. Also in this case the overall heat transfer coefficient should be replaced by the internal heat transfer convection coefficient. The overall heat transfer for this thermal system can be expressed as follows:

$$
U=\left[\frac{D_{S, o}}{D_{S, i}} \frac{1}{h_{h}}+\frac{D_{S, o} \ln D_{S, o} / D_{S, i}}{2 k_{S}}+\frac{1}{h_{c}}\right]^{-1}
$$

where $h_{h}$ is inside heat transfer coefficient for process fluid in scraper channel and $h_{c}$ is the outside heat transfer coefficient of the coolant fluid respectively. The subscript $S$ represents the scraper channel. The three ordinary differential Eq. (1) and (2) are of the kind of two boundary value problems and can be solved using Runge-KuttaFehlberg combined with the shooting method (root finding) [8] for the counter current flow pattern.

The suggest method modified by De Goede and De Jong [9] is the most acceptable to predict the heat transfer coefficient $h_{h}$ in scraper channel with present hydrodynamic boundary conditions as follows:

$$
N u_{\text {tube }}=\frac{f / 8\left(\operatorname{Re}_{a x}-1000\right) \operatorname{Pr}}{1+12.7(f / 8)^{0.5}\left(\operatorname{Pr}^{2 / 3}-1\right)}\left[1+\left(\frac{D_{e}}{L}\right)^{2 / 3}\right]\left(\frac{\operatorname{Pr}}{\operatorname{Pr}_{w}}\right)^{0.11}
$$

where $f$ is the Darcy friction factor and can be predicted from the empirical equation developed by Petukhov [9] as follows:

$$
f=\left(1.82 \cdot \log \left(R e_{a x}\right)-1.64\right)^{-2}
$$

For heat transferred to the internal stator wall for the hooted fed/solvent in the annular gab between the Stator and Rotor the proposed equation is as follows [9]:

$$
N u=N u_{\text {tube }}\left[1-0.14\left(D_{R, o} / D_{S, i}\right)^{0.6}\right]
$$

The equivalent or hydraulic diameter De in scraper channel defined by 4 times the cross sectional area divided by the wetted perimeter taken into account the area and the perimeter of the couple scraper blades.

Eq. (4) also valid to predict the $h_{c}$ for the coolant filtrate with using the corrected Nussle number for the annular space of the coolant with heat transfer from the inside pipe to the insulated outer pipe as follows [10]:

$$
N u=N u_{\text {tube }} \cdot 0.86\left(D_{o} / D_{S, o}\right)^{0.16}
$$

To avoid any confusing for the diameters in the above equations Fig. 1 showed schematically the cross sectional of the double pipe scraper surface crystallizer.

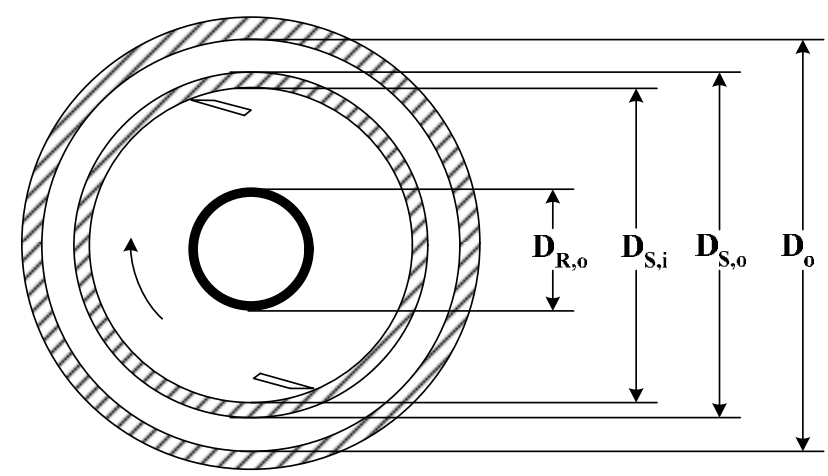

Fig. 1. Schematic of the cross section double pipe scraper surface crystallizer. 
The inner and outer pipes are made from carbon steel with length $(13.4 \mathrm{~m})$ and corresponding inner and outer diameters of $(15.24 \mathrm{~cm})$ and $(20.32 \mathrm{~cm})$ respectively. The specifications design for the train line of DPSSE $s$ and DPSSC $\mathrm{s}$ that installed in the MEK dewaxing plant consist of two bank units of the exchangers (16 pipes) for each bank are installed in series followed by two bank units of the chillers (12 pipes) for each bank.

\section{B. Kinetics of Wax Crystallization}

The particles conservation in any system is governed by the particle number continuity equation; The PBE is another transport equation to identify the particle numbers in each and every size range and accounting any changes due to particle formation [11]. The formula of the PBE for steady state considering only the nucleation and crystal growth processes is expressed as follows [7]:

$$
V \frac{\partial n}{\partial z}+\frac{\partial(n G)}{\partial L}=B^{0} . \delta\left(L-L_{c}\right)
$$

where $n=d N / d L$ is the crystal population density expressed as the number of crystal per unit size per unit volume of system, $G=d L / d t$ is the crystal growth rate, $B^{0}=d N / d t$ is the nucleation rate, $V$ is the solution mean velocity, $\mathrm{L}$ is the crystal size, and is the Dirac function to restricted the first nuclei creates with size equal to critical size $L_{c}$. The assumptions for the above PBE are; the crystals have the same shape with spherical configuration, the growth rate of the crystal is independent on the crystal size and the relative velocity of the contact crystals-solution remains constant without slip condition.

The overall crystal nucleation of the wax crystallization in the exchangers and chillers in solvent dewaxing process will be represented as follows [11]:

$$
B^{0}=k_{b} \Delta C^{b}
$$

The concentrations units will be expressed in $(\mathrm{kg}$ solute/kg solution) and using the same units in predicting the nucleation rate in Eq. (9) thus the nucleation rate constant $\mathrm{kb}$ units in this case will be in (particles $/ \mathrm{m} 3 \mathrm{~s}$ ).

In this work depended on work conducted by Beiny et al. [12] for the single particle face growth rate of the nDotriacontane (n-C32H66) dissolved in an m-xylene is adopted in this study. The experimental data is converted to fit the following linear equation:

$$
G=k_{g} \Delta C^{g}
$$

The relation above is the proposed first order growth law correlation where the exponent $\mathrm{g}$ is equal to 1 . The $k_{g}$ is the growth rate dimensional parameter characterized for the material and the process nature and its value about $\left(5.871 \times 10^{-7} \mathrm{~m} / \mathrm{s}\right)$ where predicted from the fitting of the experimental data.

The tested carbon numbers extended from $\mathrm{C}_{22}-\mathrm{C}_{36}$ and the liquid lube oil portion by represent by $\mathrm{C}_{10}$ (Decane). The nucleation rate constant and order values for n-alkanes ranged from $\mathrm{C}_{13}$ to $\mathrm{C}_{32}$ are reported by Taggart and Voogt [13] with crystallization from stagnant melts solution. The nucleation orders values for the $n$-alkanes ranged from $\mathrm{C}_{33}$ to $\mathrm{C}_{36}$ are extrapolated from the others $\mathrm{n}$-alkanes data in the reference.

The solubility of n-alkanes dissolved in solvents light nalkanes $\mathrm{C}_{10}$, aromatic, Toluene, and Ketone, MEK can be predicted [14] as follows:

$$
\begin{aligned}
& \ln \varphi_{W, i}=-\frac{\Delta H_{m}}{R}\left(\frac{1}{T}-\frac{1}{T_{m}}\right)-\frac{\Delta H_{t}}{R}\left(\frac{1}{T}-\frac{1}{T_{t}}\right)+0.5 \ln \left[\varphi_{W, i}+\left(1-\varphi_{W, i}\right) \frac{\bar{V}_{W}}{\bar{V}_{i}}\right] \\
& -0.5\left(1-\varphi_{W, i}\right)\left(1-\frac{\bar{V}_{W}}{\bar{V}_{i}}\right)-\frac{\left(1-\varphi_{W, i}\right)^{2} \bar{V}}{R T}\left({ }_{W}-{ }_{i}\right)^{2} \quad(i=O, T, M)
\end{aligned}
$$

The subscripts $O, T$, and $M$ refer to the three solvent compositions; Oil (Decane), Toluene, and MEK respectively; $R$ is the ideal gas constant $(8.3145 \mathrm{~J} / \mathrm{K} . \mathrm{mol}) ; T_{m}$ and $T_{t}$ are the melting and transition temperatures; $\Delta H_{m}$ and $\Delta H_{t}$ are the solute heat of fusion and transition respectively; is the solubility parameter.

The corresponding values of the mass fraction for the solute wax can be predicted with the alternative equation as follows:

$$
\phi_{W, i}=\frac{W_{W, i} \overline{V_{W}} M_{i}}{W_{W, i} \bar{V}_{W} M_{i}+\left(1-W_{W, i}\right) \overline{V_{i}} M_{W}}
$$

where $W_{W}$ is the mass fraction predicted as a $\mathrm{kg}$ of solute $/ \mathrm{kg}$ of the solution. $M_{W}$ and $M_{i}$ are the solute and solvent molar mass respectively.

The equilibrium solubility (saturation concentration) for the present process after inserting the volumetric flow rates of the oil feedstock and the solvents compositions (Oil, Toluene, and MEK) is as follows:

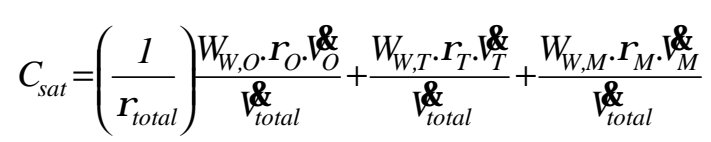

where

$\rho_{O}, \rho_{T}$, and $\rho_{M}$ are the densities of oil portion $\left(\mathrm{C}_{10}\right)$, Toluene, and MEK respectively.

The corresponding local or true concentration of the solute wax in the solution can be predicted as follows:

$$
C_{\text {true }}=\frac{W_{\text {exp }} \cdot \rho_{O} \cdot V_{O}^{\mathcal{E}}-\rho_{W} \cdot \alpha \cdot m_{3} \cdot V_{\text {total }}^{\mathcal{\alpha}}}{\rho_{\text {total }} \cdot V_{\text {total }}^{\alpha}}
$$

where $W_{\text {exp }}$ and $\rho_{W}$ are the wax mass fraction and solid wax density respectively. According to the current model the first four moment $\mathrm{s}$ differential equations can be represented as follows:

$$
V \frac{d M_{k}}{d z}=k G M_{k-1}+B^{0} L_{c}, \quad k=0,1,2,3
$$

The boundary conditions for the moments in the above equation can be taken as zero where there is no crystal exists 
in the exchanger s inlet.

The spherical critical nuclei size $L_{c}$ in Eq. 15 can be expressed in terms of the supersaturation ratio $S$ as follows [11]:

$$
L_{c}=\frac{2 \sigma_{W} v_{W}}{k_{B} T \ln S}
$$

where $k_{B}$ and $\sigma_{W}$ are the Boltzmann constant and the interfacial tension of the solid wax particles and liquid state respectively. The interfacial tension value is equal to $(0.0084 \mathrm{~N} / \mathrm{m})$ and is adopted for the whole range of $\mathrm{n}$ alkanes in this paper.

The most likely method to represent the CSD of random crystal size is by adopting the probability density function for the log-normal distribution as follows [15]:

$$
n(L)=\frac{M_{0}}{\sqrt{2 \pi}\left(\ln \sigma_{g}\right) L} \exp \left(-\frac{\ln ^{2}\left(L / L_{g}\right)}{2\left(\ln \sigma_{g}\right)^{2}}\right)
$$

where $L_{g}$ and is the geometrical average particle size (diameter) and $\sigma_{g}$ is the geometric standard deviation of the distribution. The crystals number formula can be derived by divided the both sides of Eq. (17) by the particle size $L$ as follows [16]:

$$
\frac{d N}{d \ln d}=\frac{M_{0}}{\sqrt{2 \pi}\left(\ln \sigma_{g}\right)} \exp \left(-\frac{\ln ^{2}\left(L / L_{g}\right)}{2\left(\ln \sigma_{g}\right)^{2}}\right)
$$

The $L_{g}$ and $\sigma_{g}$ quantities can be expressed in terms of the others moments as follows:

$$
M_{k}=M_{0} L_{g}^{k} \exp \left(\frac{k^{2}}{2} \ln ^{2} \sigma_{g}\right)
$$

With $k=0,1$, and 2, the expressions of $L_{g}$ and $\sigma_{g}$ as follows:

$$
\begin{gathered}
L_{g}=\frac{M_{1}}{M_{0} \exp \left(\frac{\ln ^{2} \sigma_{g}}{2}\right)} \\
\ln ^{2} \sigma_{g}=\ln \left(\frac{M_{2} M_{0}}{M_{1}^{2}}\right)
\end{gathered}
$$

The predicted moments can be detail the crystals characteristics of the wax at the exit of the scraped chillers.

\section{RESULTS AND DISCUSSION}

The experimental measurements of wax content and wax appearance temperature WAT and wax disappearance temperature WDT values are (16.917 wt \%), $42.7{ }^{\circ} \mathrm{C}$, and $46.9{ }^{\circ} \mathrm{C}$ respectively. The wax content is measured by DSC thermal analysis based on the empirical formula developed by Chen et al. [17] where the WAT and WDT are measured by cross polar microscopy CPM.

The experimental determination of the solid wax removal at the exit of the chillers is based on the DSC method measurements for both the feed and the base lube oils. The solid wax removal for three sequence operating condition are tabulated in Table I.

TABLE I: WAX REMOVAL OF THE FEEDSTOCK SAMPLE

\begin{tabular}{ccc}
\hline \multicolumn{3}{c}{ Wax removal [\%] } \\
\hline Data 1 & Data 2 & Data 3 \\
79.8 & 81.7 & 81.9 \\
\hline \hline
\end{tabular}

The predicted of the appropriate carbon number to represent the solid wax properties depends on using the solubility model through inserting the experimental WDT in Eq. (16) for the oil (Decane) alone. Table II shows the Solubility mass fraction results for three n-alkanes and the deviation with DSC wax content measurement of SN500 lube oils feedstock.

TABLE II: N-ALKANES SOLUBILITY MASS FRACTION DATA

\begin{tabular}{ccc}
\hline \hline n-alkanes & Mass fra. [wt\%] & Abs dev. [wt\%] \\
\hline $\mathrm{C}_{28}$ & 49.91 & 32.993 \\
$\mathrm{C}_{32}$ & 23.66 & 6.743 \\
$\mathrm{C}_{34}$ & 15.24 & 1.677 \\
\hline \hline
\end{tabular}

The data in Table II shows that the predicted wax content for the $\mathrm{C}_{34}$ is the close one to the feed experimental measurement.

In this study the prediction of the nucleation constant is implemented by inserting a trial value manfully until the equalization of the predicted and measured wax recoveries. By using the current model the comparison of the predicted wax recoveries with experimental measurement shows that the values of the $\mathrm{C}_{34}$ is the close one where absolute average deviation of percentage wax recovery is $(3.85 \%)$ compare with $\mathrm{C}_{32}(6 \%)$ and the $\mathrm{C}_{28}(13.5 \%)$ as shown in Table III for the three operating data.

The corresponding temperatures distribution for the nalkanes shown in Fig. 2 for the three components with absolute average deviation for $\mathrm{C}_{34}$ is $(0.21), \mathrm{C}_{33}$ is (0.247), and $\mathrm{C}_{28}$ (325) illustrate that $\mathrm{C}_{34}$ is closer one.

TABLE III: THE PERCENTAGE EXPERIMENTAL WAX RECOVERIES AT DIFFERENT N-ALKANES

\begin{tabular}{cccc}
\hline \multicolumn{4}{c}{ Wax recoveries \% } \\
\hline Exp. & $\mathrm{C}_{28}$ & $\mathrm{C}_{32}$ & $\mathrm{C}_{34}$ \\
\hline 79.8 & 79.8 & 79.8 & 79.8 \\
81.7 & 96.5 & 88.5 & 86.1 \\
81.9 & 90.1 & 83.1 & 81.2 \\
\hline \hline
\end{tabular}

The first data are used to adjust the nucleation constants and the corresponding values are $1.769 \times 10^{13}, 2.116 \times 10^{11}$, and $7.578 \times 10^{10}$ with geometric average crystal $\mathrm{L}_{\mathrm{g}}$ values about 17.9, 20.7, and 21.9 for $\mathrm{C}_{28}, \mathrm{C}_{32}$, and $\mathrm{C}_{34}$ respectively.

The model numerical results of the temperatures distribution along the DPSSE and DPSSC for SN500 feedstock in comparison with the temperatures database that 
collected from the control system panel are illustrated in Fig. 2 for the three comparison carbon number. Generally, it can be observed form the Fig. 2 that the temperatures distribution passes in two main stages. The first stage is the dissolution region (single phase) where the wax is completely dissolved in the solution. The second stage is the continuous crystallization region of the solid wax particles in the solution. The transition from one stage to another can be distinguished from the first break points of the temperatures profiles as illustrated in the Fig. 2. These points indicated the onset crystallization temperatures of the crystallization region when the supersaturation ratio exceeds the value of one. Also, it can observe from the temperatures profiles that the onset crystallization temperatures for the selected carbon numbers $\mathrm{C}_{34}$ for SN500 feed have higher values occurs at smallest distance from the inlet of the pipeline in comparison with the others n-alkanes According to the supersaturation definition in this study as the difference between the true concentration of the wax dissolved in the solution and the solubility equilibrium concentration, thus when the carbon number compound has a mass fraction higher than the measured wax content therefore this compound required more residence time and corresponding distance and more reduction of temperature to exceed the unsaturated region to the labile region with satisfy the positive value of the supersaturation. Thus, selected the carbon number which has a mass fraction close to the measured one can give a realistic behavior to distinguish firstly the onset crystallization temperature and secondly the solid wax properties essentially the equilibrium solubility behavior. The average absolute deviations between the predicted temperatures distribution and the filed data shows an absolute $\left(0.435{ }^{\circ} \mathrm{C}\right),\left(0.696{ }^{\circ} \mathrm{C}\right)$, and $\left(0.914{ }^{\circ} \mathrm{C}\right)$ for $\mathrm{C}_{34}, \mathrm{C}_{32}, \mathrm{C}_{28}$ respectively.

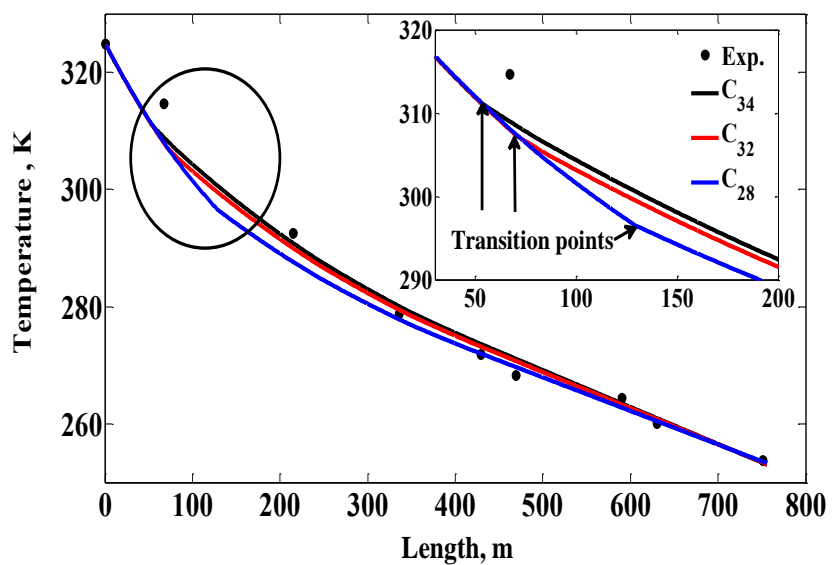

Fig. 2. Temperature distribution of the SN500 lube oil feedstock along DPSSE and DPSSC (a) data 1 (b) data 2 (c) data 3.

The wax CSD is reconstructed statistically from given moment's transformer of the PBE in the form of the lognormal distribution function of the population density (n) Fig. 3 shows the predicted CSD peaks for the n-paraffins that mention previously for the feedstock SN500. Three different peaks of the CSD's for the $\mathrm{C}_{34}, \mathrm{C}_{32}$, and $\mathrm{C}_{28}$ carbon numbers are illustrated with geometric standard deviation $\sigma \mathrm{g}$ values about $1.645,1.610$, and 1.506 for $\mathrm{C} 34, \mathrm{C}_{32}$, and $\mathrm{C}_{28}$ respectively.

The comparison implemented for the current wax CSD prediction model with the measurements results as illustrated in Fig. 4 for the wax represented compound $\mathrm{C}_{34}$ of the SN500 feedstock. Also, for the comparison purpose the predicted and measured CSD presented in the term of the particles number percentage. It can be observed the difference in the normal distribution curves of the predicted and measured CSD. Where the experimental measurements indicated mean crystal size value of $16.3 \mu \mathrm{m}$ with number percent at the peak tip around $13.8 \%$. The mean crystal size indicated a value about 31.4 and maximum number percent about $6 \%$. The CSD behaviors for the three compounds refer to the differences in the nucleation rate order data and wax mass fraction for each component.

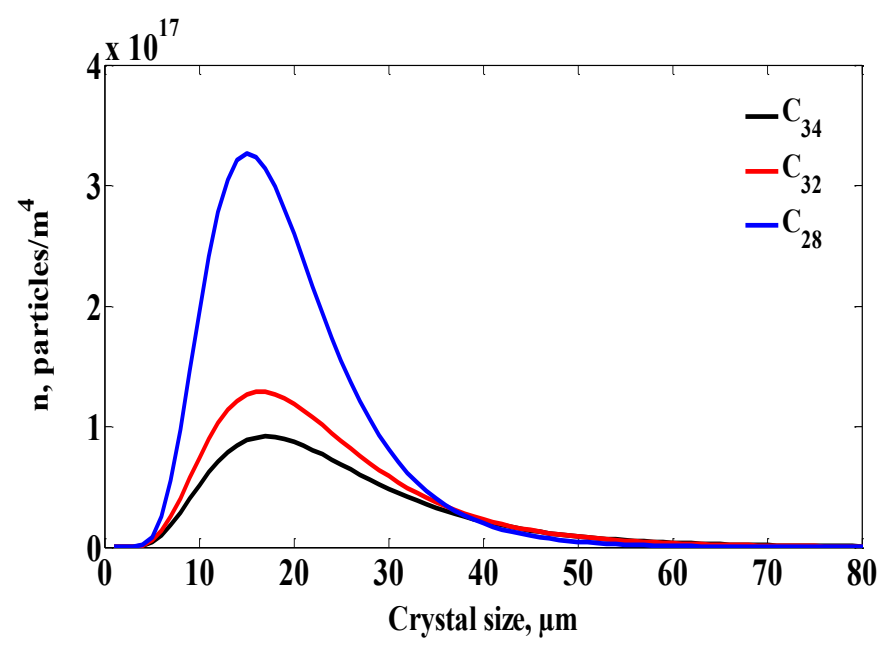

Fig. 3. Predicted CSD for different n-alkanes of SN500 feedstock database.

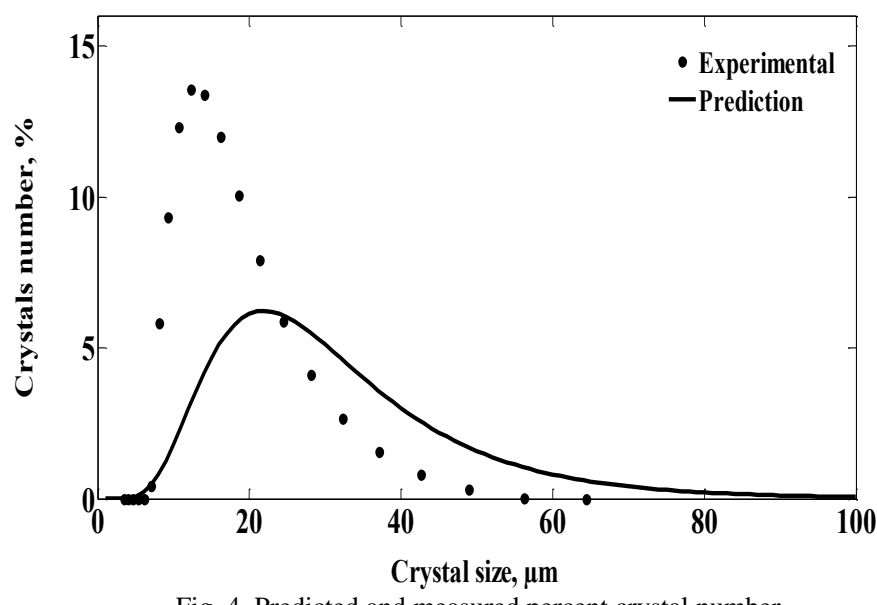

Fig. 4. Predicted and measured percent crystal number distribution.

Generally, the predicted CSD results are in the range of the experimental measurements. Although, from the above figure it can be observed that the area under the measured CSD curves are larger than the area under the predicted CSD peaks which indicated that the measured crystal numbers are greater than the predicted results. Rather than the approximate and probability of the percentage error for the experimental measurements that implemented by Mastersizer particle analyzer of the solid suspension in dispersed liquid we can specify mathematically that the main reason for this difference due to percentage error for the wax recoveries measurements techniques as these 
measurements are estimated methods even for the standards methods.

\section{CONCLUSION}

In this paper a developed model presented to predict the wax CSD had been achieved. The wax CSD predicted results shows good agreement with the experimental measurements. The measurements of the crystal size distribution were implemented by suspension particle analyzer for the extracted filtrate wax. The model is based on the available publishing data of the crystal growth nalkanes. Also, multi iteration was implemented to adjust the nucleation process by equalizing the predicted wax recovery with measurements. The comparison results for the temperature distribution and wax recoveries with plant data shows small average deviations for $\mathrm{C}_{34}$ compound via $\mathrm{C}_{32}$ and $\mathrm{C}_{28}$ components. The selection of $\mathrm{C}_{34}$ to represent wax fraction is more realistic and acceptable as this compound has small error difference between the predicted and measured mass fraction. It $s$ recommended to fit the CSD predicted results with the instant time CSD using more developing technique. This model can be applied for other types of feedstocks with different solvent injection points system.

\section{REFERENCES}

[1] A. Jr. Sequerira, Lubricant Base Oil and Wax Processing, Texaco, Inc. Port Arthur, Texas, New York, 1994.

[2] R. L. Thomas, Process Chemistry of Lubricant Base Stocks, Conventional Base Stock Production, CRC Press, 2008, ch. 6.

[3] R. J. Taylor and A. J. Mc Cormack, "Study of solvent and catalytic lube Oil dewaxing by analysis of feedstocks and products," Ind. Eng. Chem. Res., vol. 31, pp. 1731-1738, 1992.

[4] G. Lian, S. Moore, and L. Heeney, "Population balance and computational fluid dynamics modelling of ice crystallization in a scraped surface freezer," Chemical Engineering Science, vol. 61, pp. 7817-7826, 2006.

[5] H. Benkhlifa, A. H. Amamou, G. Alvarez, and D. Flick, "Modelling fluid flow, heat transfer and crystallization in a scraped surface heat exchanger," NUMB, vol. 802, pp. 163-170, 2008

[6] A. M. Bessarabov, V. P. Meshalkin, and B. E. Sel, skii, “Calculation of process of lubricating oil dewaxing in a scraper-crystallizer," Chemical and Petroleum Eng., vol. 32, no. 2, 1996.

[7] J. Li, H. He, S. Alaamri, and Y. Sul, "Modeling of a continuous crystallization process for solvent dewaxing," Separation Science and Technology, vol. 43, pp. 2023-2047, 2008.

[8] K. Muralidhar and T. Sundararajan, "Computational Fluid Flow and Heat Transfer," Alpha Science, 2nd ed, 2003.

[9] R. De Goede and E. De Jong, "Heat transfer properties of a scraped surface heat exchanger in the turbulent flow regime," Chemical Engineering Science, vol. 48, pp. 1393-1404, 1993.

[10] F. P. Incropera, A. S. Lavine, and D. P. De Witt, Fundamentals of heat and mass transfer, 7th ed., John Wiley \& Sons Incorporated, 2011.
[11] J. W. Mullin, Crystallization, 4th ed., Butterworth Heinmann, Oxford, 2001.

[12] D. H. M. Beiny, J. W. Mullin, and K. Lewtas, "Crystallization of ndotriacontane from hydrocarbon solution with polymeric additives," Journal of Crystal Growth, vol. 102, no.4, pp. 801-806, 1990.

[13] A. M. Taggart and F. Voogt, "An examination of the nucleation kinetics of n-alkanes in the homologous series $\mathrm{C} 13 \mathrm{H} 28$ to $\mathrm{C} 32 \mathrm{H} 66$, and their relationship to structural type, associated with crystallization fro stagnant melts," Langmuir, vol. 12, pp. 5722-5728, 1996.

[14] M. Claire, H. Pirson, G. Huys, and E. Vanstraelen, "New predictive equation for the solubility of solid n-alkanes in organic solvents," Ind. Eng. Chem. Res., vol. 26, pp. 447-452, 1987.

[14] K.W. Lee, H. Chen, and J. A. Gieseke, "Log-normally preserving size distribution for brownian coagulation in the Free-molecule regime," Aerosol Science and Technology," vol. 3, pp. 53-62, 1984.

[15] J. Li, H. A. Matos, and E. Gomes de Azevedo, "Two-phase homogeneous model for particle formation from gas-saturated solution processes," Journal of Supercritical Fluids, vol. 32, pp. 275286,2004

[16] J. Chen, J. Zhang, and H. Li, "Determining the wax content of crude oils by using differential scanning calorimery," Thermochimica Acta, vol. 410 , pp. 23-26, 2004.

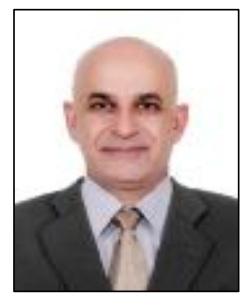

Nassir Dhamin Mokhlif is a researcher candidate at Universiti Teknologi Petronas, Malaysia. He is born in Baghdad-Iraq, in October 17, 1968 Currently in $\mathrm{PhD}$ under Mechanical Engineering discipline. He did his MSc in Mechanical Engineering, University of Tikrit-Iraq, and Bachelor of Mechanical Engineering in University of Baghdad, Iraq. He has experience in thermal design of heat exchangers, and energy flow system.

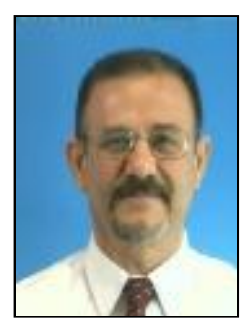

Hussain H. Al-Kayiem is an associate professor at Universiti Teknologi Petronas, Malaysia. He has served in academic and research in Iraq. He did his $\mathrm{PhD}$ in University of Bradford-UK, MSc in University of Baghdad-Iraq. He has a professional expertise in thermo-fluids, energy systems, and CFD analysis.

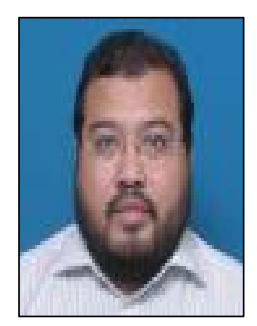

Masri Bin Baharom is an associate professor at Universiti Teknologi Petronas, Malaysia. He did his $\mathrm{PhD}$ in vehicle dynamics, University of Bradford$\mathrm{UK}, \mathrm{MSc}$ in automotive engineering, University of Leeds-UK. He has experience in automotive-vehicle and chassis design, vehicle dynamics and control. 